\title{
CONTROLE POR REALIMENTAÇÃO DE SAÍDA PARA SISTEMAS INCERTOS FORTEMENTE NÃO-LINEARES
}

\author{
Tiago Roux Oliveira* \\ tiagoroux@coep.ufrj.br
}

\author{
Alessandro Jacoud Peixoto ${ }^{\dagger}$ \\ jacoud@coep.ufrj.br
}

\author{
Liu Hsu* \\ liu@coep.ufrj.br \\ *Departamento de Engenharia Elétrica/COPPE \\ Universidade Federal do Rio de Janeiro \\ Rio de Janeiro, RJ, Brasil \\ ${ }^{\dagger}$ Departamento de Engenharia Elétrica \\ Centro Federal de Educação Tecnológica Celso Suckow da Fonseca - CEFET/RJ \\ Rio de Janeiro, RJ, Brasil
}

\begin{abstract}
Output-feedback Control for Uncertain Systems with Strong Nonlinearities

A peaking free output-feedback tracking sliding mode controller based on high-gain observers (HGO) and control activation dwell-time is introduced for a general class of uncertain single-input-single-output (SISO) nonlinear systems. Global or semi-global practical exponential stability with enlarged domains of attraction and significantly improved transient behavior are obtained. A monitoring scheme is also proposed to deal with HGO peaking induced by output exogenous disturbances.
\end{abstract}

KEYWORDS: uncertain nonlinear systems, outputfeedback, sliding mode control, peaking phenomenon.

\section{RESUMO}

Um controlador livre de peaking baseado em modos deslizantes e realimentação de saída é introduzido para o rastreamento de uma classe de sistemas não-lineares incerta utilizando observadores de alto ganho e o conceito

Artigo submetido em 31/03/2009 (Id.: 00988)

Revisado em 29/05/2009, 23/09/2009

Aceito sob recomendação do Editor Associado Prof. Alexandre Bazanella de tempo de espera na ativação do sinal de controle. Estabilidade exponencial global (semi-global) e bom comportamento transitório são obtidos. Um esquema de monitoração é também proposto para lidar com o fenômeno de peaking do HGO induzido por perturbações de saída.

PALAVRAS-CHAVE: sistemas não-lineares incertos, realimentação de saída, controle por modos deslizantes, fenômeno de peaking.

\section{INTRODUÇÃO}

Nos últimos anos o controle de sistemas incertos tem recebido uma grande atenção. Diferentes estratégias de controle foram desenvolvidas para os problemas de estabilização, regulação e rastreamento, tais como: controle por comutação (Deaecto \& Geromel, 2009), controle baseado na realimentação da derivada dos estados (Faria et al., 2009), controle em modo dual adaptativo/robusto (Cunha et al., 2009) e controle baseado em observador de alto ganho (Cunha et al., 2005; El'youssef et al., 2007; El'youssef et al., 2008).

Em especial, o rastreamento de trajetória para sistemas incertos não-lineares com grau relativo arbitrário é um problema ainda mais desafiador e não é surpresa que a 
maioria dos resultados utilizando apenas realimentação de saída impõe hipóteses restritivas nos campos vetoriais não-lineares, como por exemplo condições de crescimento particular ou a existência de uma constante de Lipschitz global.

No presente artigo, um sistema será dito fortemente não-linear se tais restrições de crescimento não precisam ser satisfeitas e, conseqüentemente, o fenômeno de escape em tempo finito não pode ser excluído a priori. Como exemplos de não-linearidades que se enquadram neste cenário estão as funções polinomiais.

Até agora, resultados de estabilidade global vem sendo obtidos apenas para uma classe restrita de plantas nãolineares e é sabido que algumas plantas muito simples com não-linearidades polinomiais não podem ser globalmente estabilizadas por realimentação de saída (Mazenc et al., 1994; Andrieu \& Praly, 2009).

Em geral, ao utilizarmos realimentação de saída, alguma estimativa do estado da planta, ou ao menos da norma do estado, é necessária. Neste sentido, observadores de alto ganho (HGO) são utilizados devido à sua robustez a incertezas paramétricas e erro de estimação arbitrariamente pequeno. Por exemplo, no contexto de linearização por realimentação de saída, HGO's têm o papel de estimar perturbações e incertezas no modelo de modo a compensá-las ou até mesmo cancelá-las. Em (Freidovich \& Khalil, 2008), é provado que o controle baseado em um HGO suficientemente rápido pode recuperar a estabilidade e o desempenho transitório do sistema nominal em malha fechada sujeito à realimentação por linearização utilizando os estados verdadeiros.

Contudo, o preço a ser pago com o HGO é a geração do fenômeno de peaking que pode levar à transientes ruins ou mesmo à instabilidade quando o peaking é transmitido para a planta (Sussmann \& Kokotović, 1991).

Oh \& Khalil (1995) e Teel \& Praly (1995) propuseram estratégias baseadas em globally bounded control (GBC), que consiste essencialmente em saturar o sinal de controle a fim de evitar os efeitos danosos do fenômeno de peaking. Entretanto, o GBC não pode garantir estabilidade global mesmo para plantas lineares instáveis em malha aberta. Com o intuito de aumentar o domínio de atração (Khalil, 2002, pp. 122), o nível de saturação do sinal de controle tem que ser aumentado. Isso por outro lado pode resultar em transitórios inaceitáveis visto que quantidades significativas do peaking continuam sendo transmitidas para a planta.

Em (Oliveira et al., 2008a; Oliveira et al., 2008b), foram propostas estratégias de controle por modos deslizantes que evitam os efeitos do peaking de acordo com a classe de sistemas não-lineares a ser controlada. No caso mais simples de sistemas com condição de crescimento linear nos estados não medidos (Oliveira et al., 2008b), obtémse estimativas para a norma do estado completo via norm observers (Sontag \& Y., 1997; Krichman et al., 2001).

De modo geral, tais estimadores da norma não são triviais de serem projetados uma vez que sua existência está ligada ao conhecimento de uma função de Lyapunov-IOSS (Input-Output-to-State Stable Lyapunov function). Deste modo, para incluir sistemas com não-linearidades fortes nos estados não medidos (e.g., polinomiais), a estratégia apresentada em (Oliveira et al., 2008a), e rediscutida aqui, obtém estimativas para a norma do estado via alto ganho, ou seja, a partir de HGOs. O peaking no sinal de controle é evitado introduzindo-se o conceito de tempo de espera ou dwelltime (Hespanha et al., 2003; De Persis et al., 2002; Freidovich \& Khalil, 2007) na ativação do sinal de controle.

Comportamento transitório uniforme, aumento do domínio de atração e menor erro residual no rastreamento de trajetória são algumas vantagens obtidas com o nosso controlador quando comparado com a abordagem GBC (Oh \& Khalil, 1997). Outra novidade aqui apresentada é a introdução de um esquema de monitoração que é utilizado como detector de peaking induzido por perturbações exógenas na saída da planta. Assim, o controlador por modos deslizantes proposto é robusto não somente à perturbação de entrada, mas também à perturbação de saída, i.e., consegue também suavizar ou até mesmo eliminar o efeito de peaking gerado por perturbações de saída. Simulações ilustram a eficácia das estratégias propostas.

\section{NOTAÇÃO E TERMINOLOGIA}

A norma Euclidiana de um vetor $x$ e a correspondente norma induzida de uma matriz $A$ são denotadas por $|x|$ e $|A|$, respectivamente. A norma $\mathcal{L}_{\infty e}$ de um sinal $x(t) \in \mathbb{R}^{n}$ é definida por $\left\|x_{t, t_{0}}\right\|:=\sup _{t_{0} \leq \tau \leq t}|x(\tau)|$; para $t_{0}=0$, a notação $\left\|x_{t}\right\|$ é adotada. O símbolo " $s$ " representa tanto a variável de Laplace quanto o operador diferencial " $d / d t$ ", de acordo com o contexto. A saída $y$ de um sistema linear e invariante no tempo com função de transferência $H(s)$ e entrada $u$ é escrita $y=H(s) u$. O termo genérico $\pi(t)$ é dito ser exponencialmente decrescente se $|\pi(t)| \leq b e^{-a t}, \forall t \geq 0$ e escalares $a, b>0$, com $b$ podendo depender das condições iniciais do sistema. As funções de classe $\mathcal{K}$ e $\mathcal{K}_{\infty}$ são definidas de acordo com (Khalil, 2002, pp. 144). As definições de ISS (Input-to-State-Stable), ISpS (Input-to-State-Stable) e OSS (Output-to-State-Stable) encontram-se em (Jiang 
et al., 1994; Sontag \& Y., 1997). A definição de Filippov para a solução de equações diferenciais com lado direito descontínuo (Filippov, 1964) e o conceito de controle equivalente estendido (Hsu et al., 2002), válido dentro e fora da superfície de deslizamento, serão utilizados ao longo do texto.

\section{FORMULAÇÃO DO PROBLEMA}

Considere a classe de sistemas incertos SISO não-lineares afins no controle e transformáveis por um difeomorfismo global na seguinte forma normal (Khalil, 2002):

$$
\begin{aligned}
\dot{\eta} & =A_{0}(\theta) \eta+\phi_{0}(x, t, \theta), \\
\dot{\xi} & =A_{\rho} \xi+B_{\rho} k_{p}(x, t, \theta)\left[u+d_{\phi}(x, t, \theta)\right], \\
y & =C_{\rho} \xi,
\end{aligned}
$$

onde $u \in \mathbb{R}$ é a entrada de controle, $y \in \mathbb{R}$ é a saída medida, $x^{T}:=\left[\eta^{T} \xi^{T}\right] \in \mathbb{R}^{n}$ é o estado não disponível com $\eta \in \mathbb{R}^{n-\rho}$ sendo referido como o estado da dinâmica interna, $\xi=\left[\begin{array}{lll}y & \dot{y} & \ldots\end{array} y^{(\rho-1)}\right]^{T} \in \mathbb{R}^{\rho}, A_{0}(\theta)$ é uma matriz constante incerta de dimensão apropriada e a tripla $\left(A_{\rho}, B_{\rho}, C_{\rho}\right)$ está na forma controlador de Brunovsky, isto é, representando uma cadeia de $\rho$ integradores (Marino \& Tomei, 1995). O vetor constante $\theta$ inclui todos os parâmetros incertos do sistema e pertence a um conjunto compacto $\Omega \subset \mathbb{R}^{p}$ tal que os limitantes para as incertezas necessários para o projeto do controlador estão disponíveis. O sistema não-linear (1) tem grau relativo arbitrário $\rho$ bem definido e constante em todo o espaço de estado $\left(\forall t\right.$ e $\forall \theta$ ), o escalar $k_{p}$ é o ganho de alta freqüência (HFG) da planta e $\phi_{0}, d_{\phi}$ são vistas como perturbações não-lineares incertas, variantes no tempo e dependentes do estado.

Sistemas nas formas output-feedback, parametric strictfeedback (Krstić et al., 1995) ou triangular (Marino \& Tomei, 1995), incluindo não-linearidades polinomiais no estado não medido ou na saída, são exemplos de classes que podem ser transformadas em (1). Deste modo, o fenômeno de escape em tempo finito não pode ser evitado a priori e assim para cada solução de (1), o máximo intervalo de tempo de definição de solução é $\left[0, t_{M}\right)$, onde $t_{M}$ pode ser finito ou infinito. Daqui em diante, $\mathcal{I}_{D}$ denota um sub-intervalo de $\left[0, t_{M}\right)$.

\subsection{Hipóteses Fundamentais}

Assume-se que $\forall(x, t) \in \mathbb{R}^{n} \times \mathbb{R}_{+}$e $\forall \theta \in \Omega$ : (H1) $\phi_{0}(x, t, \theta) \in \mathbb{R}^{n-\rho}$ é localmente Lipschitz em $x(\forall x)$ e contínua por partes em $t(\forall t)$; (H2) $d_{\phi}(x, t, \theta) \in \mathbb{R}$ é contínua em $x$ e $t$; (H3) o $\operatorname{sgn}\left(k_{p}\right)$ é constante, conhecido e $\left|k_{p}(x, t, \theta)\right| \geq \underline{k}_{p}$, onde $\underline{k}_{p}>0$ é um limitante inferior (constante) conhecido para $k_{p}$. Nossas hipóteses adicionais são:

(H4) Na dinâmica interna ou subsistema- $\eta, A_{0}$ é Hurwitz e $\left|\phi_{0}\right| \leq \epsilon|\eta|+\varphi_{0}(|\xi|, t)$, para $\epsilon \in[0, \bar{\epsilon}]$, onde $\bar{\epsilon} \geq 0$ é uma constante conhecida e $\varphi_{0}$ é uma função nãonegativa conhecida, contínua por partes e uniformemente limitada em $t$ e classe $\mathcal{K}$ em $|\xi|$.

(H5) Existe uma função classe $\mathcal{K}$ localmente Lipschitz $\varphi_{d}(|x|)$ e uma constante $c_{d} \geq 0$, ambas conhecidas, tal que $\left|d_{\phi}(x, t, \theta)\right| \leq \varphi_{d}(|x|)+c_{d}$.

De acordo com (H4), se $\bar{\epsilon}$ for suficientemente pequeno, plantas de fase mínima podem ser tratadas e é possível encontrarmos um limitante superior para $|\eta|$ por meio de um estimador da norma da dinâmica interna. Além disso, classes mais gerais poderiam ser consideradas para o subsistema- $\eta$, entretanto optou-se aqui pela classe com crescimento linear em $\eta$ apenas por simplicidade.

É importante salientar que encontrar um estimador da norma apenas para o subsistema- $\eta$ é uma tarefa mais simples do que obtermos um para o estado completo $x$ como em (Kaliora et al., 2006) ou (Hsu et al., 2003).

Note que (H5) não é restritiva visto que $d_{\phi}$ é contínua em seus argumentos. Além disso, nenhuma condição particular de crescimento é imposta à função $\varphi_{d}$. Conhecendo $\varphi_{d}$, um limitante superior para a norma da perturbação não-linear casada $d_{\phi}$ pode também ser obtido.

\subsection{Objetivo de Controle}

O objetivo é projetar uma lei de controle dinâmica $u$, via realimentação de saída, para levar o erro de rastreamento

$$
e(t)=y(t)-y_{m}(t)
$$

exponencialmente para zero ou para algum conjunto residual pequeno próximo de zero (rastreamento prático), mantendo-se todos os sinais do sistema em malha fechada limitados, independentemente das incertezas.

A trajetória desejada $y_{m}(t)$ é gerada pelo seguinte modelo de referência:

$$
y_{m}=M(s) r=\frac{k_{m}}{L(s)\left(s+a_{m}\right)} r, \quad k_{m}, a_{m}>0,
$$

onde $r(t)$ é assumida contínua por partes, uniformemente limitada e o polinômio Hurwitz $L(s)$ é dado por

$$
L(s):=s^{\rho-1}+a_{\rho-2} s^{\rho-2}+\ldots+a_{0} .
$$




\subsection{Dinâmica do Erro de Rastreamento}

Considere a realização mínima de $M(s)$ em (3) dada por:

$$
\dot{\xi}_{m}=A_{m} \xi_{m}+B_{m} k_{m} r, \quad y_{m}=C_{m} \xi_{m},
$$

onde $\xi_{m}^{T}:=\left[\begin{array}{llll}y_{m} & \dot{y}_{m} & \ldots & y_{m}^{(\rho-1)}\end{array}\right], B_{m}:=B_{\rho}, C_{m}:=C_{\rho} \mathrm{e}$ $A_{m}:=A_{\rho}+B_{\rho} K_{m}$, com $K_{m}$ obtido a partir dos coeficientes do polinômio característico de $M(s)$.

Agora, considerando-se a dinâmica- $\xi$ dada em (1) e substituindo-se $u$ por $u+K_{m} \xi / k_{p}-K_{m} \xi / k_{p}$, tem-se

$$
\dot{\xi}=A_{m} \xi+B_{m} k_{p}\left[u-K_{m} \xi / k_{p}+d_{\phi}\right], \quad y=C_{m} \xi .
$$

A partir de (5) e (6), o estado $x_{e}:=\xi-\xi_{m}$ e o erro de rastreamento $e$ satisfazem

$$
\dot{x}_{e}=A_{m} x_{e}+k_{p} B_{m}[u+d], \quad e=C_{m} x_{e},
$$

onde a perturbação equivalente de entrada é definida por

$$
d(x, t, \theta):=-k_{m} r / k_{p}-K_{m} \xi / k_{p}+d_{\phi}(x, t, \theta) .
$$

\section{CONTROLE POR REALIMENTAÇÃO DE SAÍDA E MODOS DESLIZANTES}

Quando apenas y está disponível para realimentação, a superfície de deslizamento pode ser escolhida como

$$
\begin{aligned}
\hat{\sigma} & :=S \hat{x}_{e}=0, \\
S & :=\left[\begin{array}{llll}
a_{0} & \ldots & a_{\rho-2} & 1
\end{array}\right], \\
\hat{x}_{e} & :=\hat{\xi}-\xi_{m},
\end{aligned}
$$

onde $a_{0}, \ldots, a_{\rho-2}$ é definido em (4), tal que $\left(A_{m}, B_{m}, S\right)$ com estado $x_{e}$ em (7) seja estritamente real positivo (strictly positive real - SPR) (Marino \& Tomei, 1995) e $\hat{\xi}$ é uma estimativa de $\xi$ fornecida pelo HGO devido sua robustez à perturbações e incertezas paramétricas. A lei de controle $u$ é definida por

$$
u=-\left[\operatorname{sgn}\left(k_{p}\right)\right] \varrho(\chi, t) \operatorname{sgn}(\hat{\sigma}(t)),
$$

onde $\chi(t)$ é uma função escalar não-negativa absolutamente contínua, obtida a partir de sinais disponíveis e que limita superiormente a norma do estado da planta $|x|$, modulo termos exponencialmente decrescentes.

Deste modo, é desejável obter um limitante em norma livre de peaking $\chi$ tal que a a função de modulação $\varrho(\chi, t)$ satisfaça

$$
\varrho(\chi, t) \geq|d(x, t, \theta)|+\delta,
$$

a menos de termos exponencialmente decrescentes, onde $\delta>0$ é uma constante arbitrária pequena. Diferentemente do caso de grau relativo um, a desigualdade (11) não é suficiente para alcançarmos rastreamento global ou semi-global devido a perturbações provenientes da estimação inexata do HGO. Entretanto, sendo o erro de estimação dado por

$$
\tilde{x}_{e}:=x_{e}-\hat{x}_{e}=\xi-\hat{\xi},
$$

o seguinte lema para realimentação de saída pode ser enunciado.

\section{Lema 1 (Propriedade ISS de $\left|\tilde{x}_{e}\right|$ para $x_{e}$ )}

Considere a dinâmica que governa $x_{e}$ em (7) com saída $\hat{\sigma}=S x_{e}-S \tilde{x}_{e}, u$ dado em (10), @ satisfazendo (11) e d em (8). Então, (7) é ISS com respeito a $\tilde{x}_{e}$ e a seguinte desigualdade se verifica

$$
\left|x_{e}(t)\right|,|e(t)| \leq k_{e}\left|\tilde{x}_{e}(t)\right|+\pi_{e}, \quad \forall t \in \mathcal{I}_{D},
$$

onde $\pi_{e}$ denota um termo que decai exponencialmente $e$ $k_{e}>0$ é uma constante apropriada.

Prova: Ver Apêndice A.

Com base no Lema 1, o objetivo é fornecer uma estimativa $\hat{\xi}$ por meio de um HGO com ganho apropriado tal que a norma do erro de observação $\left|\tilde{x}_{e}(t)\right|$ seja arbitrariamente pequena, assegurando rastreamento (prático) global ou semi-global.

No esquema proposto e representado na Fig. 1, um eventual peaking em $\hat{\sigma}$ é bloqueado pela função $\operatorname{sgn}(\cdot)$ em (10) e o sinal de controle $u$ é livre de peaking visto que $\chi$ é implementado utilizando-se apenas sinais disponíveis bem condicionados, isto é, sem peaking. A seguir, é dada uma breve descrição do HGO e da estratégia livre de peaking utilizada para obtermos $\chi$.

\section{OBSERVADOR DE ALTO GANHO}

A estimativa $\hat{\xi}$ para $\xi$ em (1) é fornecida pelo HGO:

$$
\dot{\hat{\xi}}=A_{\rho} \hat{\xi}+k_{p}^{\text {nom }} B_{\rho} u+H_{\mu} L_{o} C_{\rho}(\xi-\hat{\xi})
$$

onde $L_{o}:=\left[l_{1} \ldots l_{\rho}\right]^{T}$ e $H_{\mu}:=\operatorname{diag}\left(\mu^{-1}, \ldots, \mu^{-\rho}\right)$. O ganho do observador $L_{o}$ é tal que $N(s)=s^{\rho}+l_{1} s^{\rho-1}+\ldots+l_{\rho}$ é Hurwitz e $k_{p}^{\text {nom }}$ é um valor nominal para $k_{p}$. Uma vez que deseja-se que as incertezas e perturbações tenham efeitos desprezíveis em $\hat{x}_{e}(9)$, a norma de $H_{\mu}$ deverá ser grande (alto ganho), impondo-se assim que o parâmetro constante $\mu$ seja suficientemente pequeno.

\subsection{Fenômeno de Peaking}

Como é sabido, as estimativas do HGO podem apresentar peaking à medida que $\mu \rightarrow 0$ (Sussmann \& Kokotović, 1991). De fato, o erro de estimação $\tilde{x}_{e}$ (12) irá 


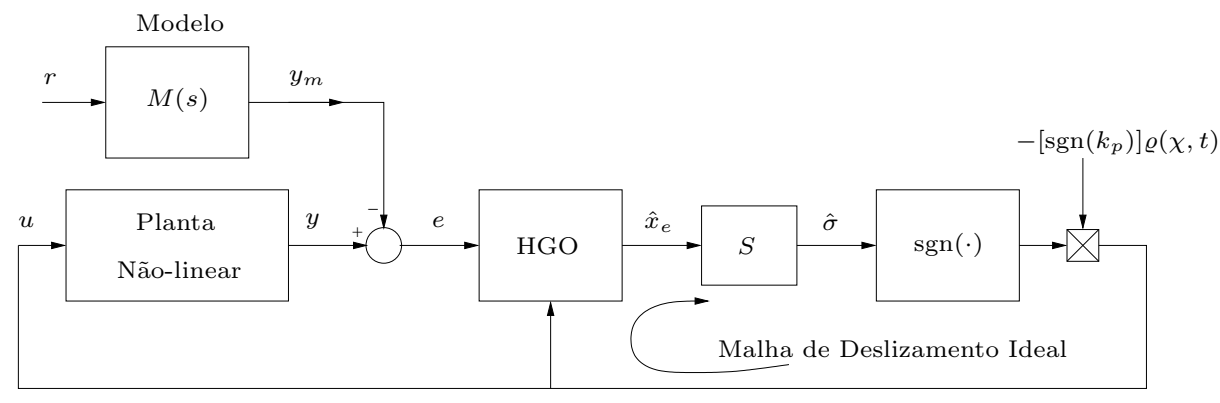

Figura 1: Controlador por modos deslizantes e realimentação de saída.

conter termos transientes da forma $\left(a / \mu^{b}\right) e^{-c t / \mu}$, onde $a, b, c>0$ são constantes dependentes dos parâmetros da planta. Em poucas palavras, esses termos eventualmente irão exibir um comportamento transitório impulsivo quando $\mu \rightarrow 0$, onde os picos transientes atingem valores da ordem de $\mathcal{O}(1 / \mu)$ antes de decaírem rapidamente para zero.

Contudo, o fenômeno de peaking pode ser superado utilizando-se o conceito de tempo de extinção de pico $\left(t_{e}\right)$ (Cunha et al., 2005), onde $t_{e}(\mu)$ é definido como a solução de $\left(a / \mu^{b}\right) e^{-c t_{e} / \mu}=1$, para cada valor de $\mu \in(0,1]$. Na Seção 6, este conceito será crucial na formulação da lei de controle livre de peaking.

O tempo de extinção de pico é análogo ao tempo de retorno definido em (Boyd et al., 1994, Sec. 5.2.3), onde o problema de encontrar limitantes superiores para este foi formulado. Apesar de $t_{e}$ ser desconhecido, utilizando os limitantes superiores conhecidos para os parâmetros incertos da planta $(\theta \in \Omega)$, pode-se obter um limitante superior conhecido $\bar{t}_{e}(\mu) \in \mathcal{K}$ tal que $t_{e}(\mu) \leq \bar{t}_{e}(\mu)$ (Cunha et al., 2005).

\subsection{Dinâmica do Erro do Observador}

Assim como em (Oh \& Khalil, 1997), a seguinte transformação de coordenadas será utilizada em (12):

$$
\zeta:=T_{\mu} \tilde{x}_{e}, \quad T_{\mu}:=\left[\mu^{\rho} H_{\mu}\right]^{-1} .
$$

Note que: (i) $T_{\mu}\left(A_{\rho}-H_{\mu} L_{o} C_{\rho}\right) T_{\mu}^{-1}=\frac{1}{\mu} A_{o}$ e (ii) $T_{\mu} B_{\rho}=$ $B_{\rho}$, onde $A_{o}:=A_{\rho}-L_{o} C_{\rho}$. Assim sendo, a partir da dinâmica de $\xi$ em (1), de (12), (13) e (14), tem-se

$$
\mu \dot{\zeta}=A_{o} \zeta+k_{p} B_{\rho}[\mu \nu]
$$

$\operatorname{com} \nu:=\left[\kappa u+d_{\phi}\right]$ e $\kappa=\left(k_{p}-k_{p}^{\text {nom }}\right) / k_{p}$.

\section{ESTRATÉGIA PARA EVITAR PEAKING VIA DWELL-TIME}

Neste artigo, assim como em (Oh \& Khalil, 1995; Oh \& Khalil, 1997), utiliza-se as estimativas do HGO (com peaking) não apenas para definir a superfície de deslizamento, mas também para obtermos $\chi$ e uma função de modulação $\varrho(\chi, t)$ adequada.

Em (Oh \& Khalil, 1995; Oh \& Khalil, 1997), o fenômeno de peaking é evitado utilizando-se funções de saturação na lei de controle (GBC). Como conseqüência, o "esforço" de controle necessário deve ser primeiramente estimado de modo a podermos ajustar corretamente o nível de saturação e garantirmos assim a estabilidade semi-global.

O maior problema desta abordagem é que para estendermos o domínio de atração, é necessário aumentarmos o nível de saturação. Deste modo, uma energia considerável do peaking é ainda transmitida para a planta, o que resulta em degradação do comportamento transitório (principalmente para condições iniciais pequenas) e contração do domínio de atração.

Inspirados pelos recentes desenvolvimentos em esquemas baseados no controle supervisório e com chaveamento (Hespanha et al., 2003; De Persis et al., 2002; Freidovich \& Khalil, 2007), propõe-se aqui uma nova estratégia baseada no conceito de tempo de espera ou dwell-time de modo a lidar com os problemas induzidos pelo peaking comentados anteriormente.

\subsection{Observador com Dwell-Time}

A idéia chave consiste em combinar as estimativas de alto ganho do HGO com uma estratégia apropriada, baseada no conceito de dwell-time, de forma a obter um limitante em norma $\chi$ livre de peaking para o estado da planta $x$. Neste sentido, aplica-se as estimativas do HGO apenas depois de um certo tempo $\tau_{D}$ (dwell-time) 
que será grande o bastante para permitir que o transiente devido ao peaking no HGO acomode-se, e pequeno o suficiente para garantir que as trajetórias do sistema não deixem um determinado conjunto compacto, evitando-se assim o escape em tempo finito.

Para todo $t \in\left[0, \tau_{D}\right)$, o sinal de controle é nulo $(u=0)$. Uma vez que o tempo de extinção de pico $t_{e}$ satisfaz $t_{e}(\mu) \leq \bar{t}_{e}(\mu), \operatorname{com} \bar{t}_{e}(\mu) \in \mathcal{K}$ conhecido, então escolhendose (Oliveira et al., 2008a; Oliveira et al., 2008b)

$$
\tau_{D}:=\bar{t}_{e}(\mu),
$$

existe $\mu_{1} \in(0,1]$ suficientemente pequeno, tal que evitase o escape em tempo finito durante o dwell-time para todo $0<\mu \leq \mu_{1}$.

De fato, para qualquer condição inicial dada e $u=0$ em (1), se $x(t)$ eventualmente escapar em algum tempo finito $t_{M}$, então $t_{M}$ necessariamente será independente de $\mu$. Além disso, a partir de (13) pode-se concluir o mesmo para $\hat{\xi}$ pois $A_{\rho}-H_{\mu} L_{o} C_{\rho}$ é uma matriz Hurwitz. Portanto, reduzindo $\mu$ assegura-se que a norma do estado aumentado $x_{a}(t):=\left[\begin{array}{ll}x^{T} & \hat{\xi}^{T}\end{array}\right]^{T}$ é uniformemente limitada no intervalo $\left[0, \tau_{D}\right)$, após o qual, os transitórios do HGO (peaking) foram extintos.

No que segue, seja $t^{*} \in\left[\tau_{D}, t_{M}\right)$ o primeiro instante de tempo tal que o estado aumentado $x_{a}(t)$ sai de uma bola compacta dada $\mathcal{B}:=\left\{x_{a}:\left|x_{a}(t)\right| \leq R\right\}$ de raio $R>$ $\left|x_{a}\left(\tau_{D}\right)\right|$ e considere o sub-intervalo $\mathcal{I}_{D}:=\left[\tau_{D}, t^{*}\right)$.

\section{Observação 1 [Projeto Prático do Dwell-Time]}

Para simplificar a implementação do tempo de espera (dwell-time), pode-se utilizar uma constante $\tau_{D}$ apropriada em vez do cálculo exato de $\bar{t}_{e}$. Note que, é sempre possível escolher constantes $\bar{\tau}_{D}$ e $\mu$, suficientemente pequenas, tais que para $\tau_{D}=\bar{\tau}_{D}$, o peaking e o escape em tempo finito são evitados durante o intervalo de tempo de espera, ou seja, $\tau_{D}$ satisfaz a desigualdade $\bar{t}_{e}(\mu) \leq \tau_{D}<t_{M}$.

\subsection{Limitante para a Norma do Estado}

Devido à propriedade de alto ganho do HGO, pode-se mostrar que: enquanto o estado aumentado $x_{a}$ permanece dentro da bola $\mathcal{B}, \forall t \in\left[\tau_{D}, t^{*}\right)$, o erro de observação $\tilde{x}_{e}$ (12) pode ser feito arbitrariamente pequeno reduzindo-se o parâmetro $\mu$ do HGO. De fato, se $\mu$ é suficientemente pequeno tal que $\tau_{D}(\mu) \in\left[0, t^{*}\right)$, tem-se que $\forall t \in\left[\tau_{D}, t^{*}\right)$ [vide (Oh \& Khalil, 1995, Lemma 1) e (Oliveira et al., 2008a, Proposition 1)]:

$$
\left|\tilde{x}_{e}(t)\right|=|\xi(t)-\hat{\xi}(t)| \leq \mathcal{O}(\mu),
$$

$$
|x(t)|-\pi(t) \leq|\bar{\eta}(t)|+|\hat{\xi}(t)|+\Delta:=\chi(t),
$$

onde $\hat{\xi}$ é a estimativa do HGO para $\xi, \Delta>0$ é uma constante que majora o efeito do erro de estimação $\mathcal{O}(\mu)$ em $(17)$ e $\bar{\eta}(t)=\frac{c_{0}}{s+\lambda_{0}} \varphi_{0}(|\hat{\xi}(t)|, t)$ é o estimador da norma projetado para a dinâmica- $\eta$ em (1) com constantes apropriadas $c_{0}, \lambda_{0}>0$ e $\varphi_{0}$ dada em (H4), considerando-se $\bar{\epsilon}$ em (H4) suficientemente pequeno [vide (Hsu et al., 2003, Lemma 2)]. Note que, para provarmos (18) utilizamos a relação $|x(t)| \leq|\eta(t)|+|\xi(t)|$ e a desigualdade $|\eta(t)| \leq|\bar{\eta}(t)|$, válida $\forall t \in\left[\tau_{D}, t^{*}\right)$, modulo o termo exponencialmente decrescente $\pi(t)$.

\subsection{Função de Modulação}

A partir de (H5), (8) e (18), pode-se escrever $|d| \leq \hat{d}+\pi_{d}$,

$$
\hat{d}(t):=\left(k_{m}|r|+\left|K_{m}\right| \chi\right) / \underline{k}_{p}+\varphi_{d}(2 \chi)+c_{d},
$$

notando-se que $\underline{k}_{p}>0$ é um limitante inferior conhecido para $k_{p}$ e $\varphi_{d}(\chi+\pi) \leq \varphi_{d}(2 \chi)+\varphi_{d}(2 \pi)$, onde $\pi_{d}(t)=\varphi_{d}(2 \pi)$ é um termo exponencialmente decrescente.

A função de modulação livre de peaking $\varrho(\chi, t)$ pode então ser obtida tal que (11) seja válido $\forall t \in\left[\tau_{D}, t^{*}\right)$ :

$$
\varrho(\chi, t)= \begin{cases}0 & , \forall t \in\left[0, \tau_{D}\right) \\ \hat{d}(t)+\delta & , \text { caso contrário } .\end{cases}
$$

No próximo teorema é demonstrado que mesmo com $u:=0, \forall t \in\left[0, \tau_{D}\right)$, o sistema em malha fechada não pode ter escape em tempo finito e além disso, o rastreamento global/semi-global de trajetória é garantido.

\section{ANÁLISE DE ESTABILIDADE}

A fim de considerar todas condições iniciais envolvidas no sistema do erro (7) e (14)-(15), seja:

$z^{T}(t):=\left[z^{0}(t), x_{e}^{T}(t), \tilde{x}_{e}^{T}(t)\right], \quad z^{0}(t):=\left[\eta^{T}(0) x_{\varrho}^{T}(0)\right] e^{-\gamma t}$,

onde $\gamma>0$ é uma constante genérica e $z^{0}$ denota o estado transiente (Hsu et al., 2002) devido às condições iniciais dos sistemas estáveis correspondentes à dinâmica- $\eta$ e aos filtros usados na função de modulação. O resultado principal é agora estabelecido.

Teorema 1 Considere a planta (1), a lei de controle dada por (10) e (20), o observador de alto ganho (13) e o modelo de referência em (3). Assuma que as hipóteses (H1)-(H5) sejam satisfeitas. Então, para $\mu \in(0,1]$ suficientemente pequeno, o sistema completo do erro (7) e (15) com estado $z(t)$ é globalmente ou semi-globalmente exponencialmente estável com respeito a um conjunto residual pequeno de ordem $\mathcal{O}(\mu)$ independente das condições iniciais. Além disso, todos os sinais do sistema em malha fechada são uniformemente limitados. 
Prova: Ver Apêndice B.

Corolário 1 [Malha de Deslizamento Ideal]

Adicionalmente as hipóteses do Teorema 1 , se $\varrho \geq$ $\left|K_{m}\right|\left|\xi_{m}\right|+\left|k_{m}\right||r|+\delta \operatorname{com} \delta>0$, então o modo deslizante $\hat{\sigma}(t) \equiv 0$ é alcançado em tempo finito e o fenômeno de chattering (Edwards \& Spurgeon, 1998) no sinal de controle é evitado (vide Fig. 1).

\section{Prova: Ver Apêndice C.}

Observação 2 [Ausência de Peaking]

Uma vez que o peaking não é transmitido para o estado da planta $x$, pode-se concluir que $x_{e}$ é livre de peaking notando-se que $x_{e}$ em (7) é ISS com respeito a $[u+d]$ e a lei de controle $u$ (10) é livre de peaking por definição.

Observação 3 [Conjuntos Residuais Menores]

O conjunto residual no Teorema 1 é de ordem $\mathcal{O}(\mu)$, enquanto que na abordagem GBC (Oh \& Khalil, 1995; Oh \& Khalil, 1997) esse conjunto é de ordem $\mathcal{O}(\sqrt{\mu})$.

Observação 4 [Domínio de Atração]

No Teorema 1, o sistema de controle é semi-global em apenas um único parâmetro $(\mu)$, enquanto que na abordagem GBC (Oh \& Khalil, 1995; Oh \& Khalil, 1997) mais um parâmetro precisa ser ajustado: o nível de saturação. Conjectura-se aqui que essa dependência em dois parâmetros é a razão para os menores domínios de atração observados nas simulações com o GBC na Seção 9. Como esperado, a saturação pode reduzir o domínio de atração. De fato, aumentando-se o nível de saturação, enquanto $\mu$ é fixado, o domínio de atração com o GBC é reduzido devido aos altos níveis de peaking ainda transmitidos para a planta.

\section{Observação 5 [Transitório e Ruído de Medição]}

A fim de recuperar (aumentar) o domínio usando o GBC, é necessário reduzir $\mu$, isto é, aumentar o ganho do HGO. Entretanto, enquanto o domínio de atração é aumentado, o comportamento transitório é degradado devido: (i) às maiores amplitudes do peaking transmitidas para a planta permitidas pelos níveis maiores de saturação, e (ii) a uma maior sensibilidade à ruído de medição que é observada em razão do maior ganho requerido no HGO.

\section{ESQUEMA PARA MONITORAÇÃO DE PEAKING}

Para implementar a estratégia baseada em HGO e dwelltime, é necessário saber o instante de tempo inicial de modo a manter o sinal de controle igual a zero durante o período $\tau_{D}$. Enquanto essa tarefa parece fácil quando o sistema é inicializado, isto será difícil de ser realizado se o sistema estiver sujeito a perturbações exógenas que causem mudanças abruptas na saída da planta (por exemplo, devido a falhas intermitentes de sensores (Li \& Tao, 2009) ou sensores de natureza binária (Yin et al., 2009) utilizado em aplicações de comunicação móvel) e, conseqüentemente, induzam outro peaking no HGO após o período de dwell-time inicial.

A fim de lidar com o fenômeno de peaking após essa fase inicial, um esquema de monitoração é proposto para detecção do mesmo. Se o sistema é livre de perturbações exógenas de saída, $|\xi|+\mathcal{O}(\mu)$ é um limitante superior natural para $|\hat{\xi}|$ obtido a partir de (17), válido $\forall t \geq \tau_{D}$. Além disso, como estabelecido no Teorema 1, $\xi$ tende para $\xi_{m}$ exponencialmente. Neste caso, existe um tempo finito $t_{a} \geq 0$ tal que $|\hat{\xi}| \leq\left|\xi_{m}\right|+\mathcal{O}(\mu), \forall t \geq t_{a}$. Assim, a seguinte função de monitoração $\Phi$ pode ser definida

$$
|\hat{\xi}(t)|<\left|\xi_{m}(t)\right|+\alpha:=\Phi(t),
$$

onde $\alpha>\mathcal{O}(\mu)>0$ é uma constante de projeto apropriada tal que $\Phi$ seja um limitante em norma para $|\hat{\xi}|, \forall t \geq \tau_{D}$. Lembrando que (22) é válida somente se o sistema é livre de perturbações de saída, utiliza-se $\Phi$ como um benchmark para detectar se a desigualdade em (22) é violada. Assim, o instante de detecção $\bar{t}_{i}$ é definido por

$\bar{t}_{i+1}:= \begin{cases}\min \left\{t>\bar{t}_{i}+\tau_{D}:|\hat{\xi}(t)| \geq \Phi(t)\right\}, & \text { se existir, } \\ +\infty, & \text { caso contrário, }\end{cases}$

onde $i \in \mathcal{J}:=\{0,1,2, \ldots\}$ e $\bar{t}_{0}:=0$.

Assim, (22)-(23) em conjunto com a estratégia de dwelltime formam um esquema de monitoração para detectar e evitar o fenômeno de peaking no sinal de controle também válido $\forall t \geq \tau_{D}$.

Em poucas palavras, o sinal de controle aplicado a planta é igual a zero durante o intervalo $\left[\bar{t}_{i}, \bar{t}_{i}+\tau_{D}\right)$ e é dado por (10) e (20) caso contrário. Os passos para implementar o esquema de monitoração são dados no algoritmo a seguir.

\section{Algoritmo para Monitoração de Peaking}

- Passo 1. Defina o instante de detecção inicial $\bar{t}_{0}:=0$, o conjunto indexado $\mathcal{J}:=\{0,1,2, \ldots\}$ e o valor inicial para $i \in \mathcal{J}$, isto é, $i:=0$.

- Passo 2. Para $t \geq \bar{t}_{i}$, feche a malha de controle com $u$ definido por (10) e $\varrho(\chi, t)$ em (20), alterando o instante inicial de 0 para $\bar{t}_{i}$. Cheque continuamente (22), $\forall t \geq \bar{t}_{i}+\tau_{D}$.

- Passo 3. Quando um novo peaking for detectado de acordo com (23), faça $i:=i+1$ e volte para o Passo 2 . 
O esquema de monitoração proposto é aplicável à classes de perturbações de saída em que os efeitos de peaking desapareçam após o intervalo selecionado de dwell-time e tais que as ocorrências de peaking, possivelmente múltiplas dentro do dwell-time, sejam separadas por períodos de tempo suficientemente grandes entre elas. Deste modo, os efeitos do dwell-time são absorvidos pelas propriedades de estabilidade da lei de controle por modos deslizantes durante o período sem peaking.

No Exemplo 2 da próxima seção, o algoritmo para detecção de peaking é testado com perturbações de saída do tipo pulso. No futuro, será desejável caracterizar classes mais gerais de perturbações de saída que possam ser incluídas na abordagem proposta.

\section{EXEMPLOS E SIMULAÇÕES}

Nesta seção são apresentados dois exemplos: um para ilustrar a aplicabilidade e outro para ilustrar o desempenho do esquema de controle livre de peaking proposto. O primeiro é um exemplo prático que trata de um mancal magnético e o segundo ilustra um caso em que a presença de peaking no sinal de controle pode prejudicar o transitório, levar à instabilidade (contração do domínio de atração) e ao escape em tempo finito.

Exemplo 1 [Aplicação Prática] Considere o seguinte modelo de um grau de liberdade de um mancal magnético extraído de (Arcak \& Kokotović, 2001)

$$
\dot{\bar{x}}_{1}=\bar{x}_{2}, \quad \dot{\bar{x}}_{2}=\bar{x}_{3}+\bar{x}_{3}\left|\bar{x}_{3}\right|, \quad \dot{\bar{x}}_{3}=u, \quad y=\bar{x}_{1},
$$

onde o objetivo de controle é estabilizar o sistema em $\bar{x}=0$ utilizando-se a posição do rotor $\bar{x}_{1}$, enquanto que a velocidade $\bar{x}_{2}$ e o fluxo magnético $\bar{x}_{3}$ não estão disponíveis para realimentação. A dificuldade principal é o termo quadrático no estado não medido $\bar{x}_{3}$. Entretanto, o objetivo de controle pode ser alcançado com o esquema proposto neste artigo simplesmente notando-se que o sistema em questão pode ser levado para a forma normal (1), sem dinâmica interna e sem perturbação de entrada $\left(d_{\phi} \equiv 0\right)$, utilizando a seguinte transformação de coordenadas $T(\bar{x})=\left[\begin{array}{lll}\bar{x}_{1} & \bar{x}_{2} & \bar{x}_{3}\left(1+\left|\bar{x}_{3}\right|\right)\end{array}\right]$. De fato, após a transformação, tem-se que

$$
\dot{\xi}_{1}=\xi_{2}, \quad \dot{\xi}_{2}=\xi_{3}, \quad \dot{\xi}_{3}=k_{p}\left(\bar{x}_{3}\right) u, \quad y=\xi_{1},
$$

onde o ganho de alta freqüência $k_{p}=1+2\left|\bar{x}_{3}\right|$ é positivo e não se anula $\left(\underline{k}_{p}=1\right)$. Sendo assim, pelo Teorema 1 , ao menos a estabilização $(r(t) \equiv 0)$ semi-global é assegurada.
Exemplo 2 [Exemplo Acadêmico] Considere o sistema não-linear já representado na forma normal (1) sem dinâmica- $\eta$ e com grau relativo $\rho=3$ :

$$
\dot{\xi}_{1}=\xi_{2}, \quad \dot{\xi}_{2}=\xi_{3}, \quad \dot{\xi}_{3}=k_{p}\left[u+\theta \xi_{3}^{3}\right], \quad y=\xi_{1},
$$

onde o HFG satisfaz $k_{p}>1,|\theta| \leq 2$ e $d_{\phi}=\theta \xi_{3}^{3}$ em (1). As simulações foram realizadas $\operatorname{com} \theta=1$ e $k_{p}=1.5$ como os parâmetros reais da planta. O modelo de referência é escolhido com $M(s)=\frac{1}{(s+1)^{3}}, K_{m}=[-1-3-3]^{T}$ e $r(t)=2 \sin (\pi t)$. Os parâmetros do HGO são: $\mu=0.01$, $k_{p}^{n o m}=1, N(s)=(s+5)^{3}$ e $L_{o}=\left[\begin{array}{lll}15 & 75 & 125\end{array}\right]^{T}$.

A função de modulação (20) é calculada $\operatorname{com} \delta=0.1 \mathrm{e}$ o limitante para $d$ em (8) é dado por

$$
\hat{d}(t)=|r|+|y|+2\left(\left|\hat{\xi}_{3}\right|+\Delta\right)+\left[2\left(\left|\hat{\xi}_{3}\right|+\Delta\right)\right]^{3},
$$

onde $\Delta=1$ é adicionado a $\left|\hat{\xi}_{3}\right|$ de forma a majorar o erro de estimação de ordem $\mathcal{O}(\mu)(17)$ do HGO após a fase de peaking.

Neste exemplo, o sistema tem uma não-linearidade forte (cúbica) no estado não medido $\xi_{3}$. Assim, nenhuma das estratégias via realimentação de saída existentes na literatura podem estabilizar globalmente ou garantir rastreamento global ao sistema acima (Andrieu \& Praly, 2009).

Além disso, se o peaking das estimativas do HGO fosse transmitido completamente para a planta, um escape em tempo finito teria acontecido. De fato, considerando o estado inicial $\xi(0)=\left[\begin{array}{lll}1 & 0 & 0\end{array}\right]^{T}$, o escape em tempo finito ocorre em $t=0.262 \mathrm{~s}$ (curvas não mostradas). Contudo, a estratégia de controle livre de peaking baseada em HGO e dwell-time com $\tau_{D}=10 \mu=0.1$ resulta em um excelente rastreamento das trajetórias do modelo e elimina o escape em tempo finito para as mesmas condições iniciais, vide Fig 2. Um modo alternativo para se evitar o peaking consiste em aplicar a estratégia de controle GBC (Oh \& Khalil, 1997) utilizando-se um nível de saturação apropriado $u_{\text {sat }}$ que considere o esforço de controle necessário. Entretanto, como esperado, a saturação pode reduzir o domínio de atração. Quando o parâmetro $\mu$ é fixado e aumentamos $u_{\text {sat }}$, esse domínio é reduzido devido aos maiores níveis de peaking transmitidos para a planta. De fato, para $\xi(0)=\left[\begin{array}{lll}\xi_{1}(0) & 0 & 0\end{array}\right]^{T}$ (todas as demais condições do sistema em malha fechada foram consideradas nulas), $\mu=0.01$ e $u_{\text {sat }}=500$, o rastreamento é alcançado apenas com $\left|\xi_{1}(0)\right| \leq 2$. Por outro lado, o domínio de atração em nosso esquema é consideravelmente maior $\left(\left|\xi_{1}(0)\right| \leq 8\right)$ para esse mesmo $\mu^{1}$. Além disso, quando $\xi_{1}(0)=2$, nosso esquema apresenta uma máxima amplitude de controle igual a 500. Deste modo, para garantirmos uma comparação justa entre os dois controladores,

\footnotetext{
${ }^{1}$ Para obtermos o domínio $\left|\xi_{1}(0)\right| \leq 8$ com o controlador GBC, $\mu$ deveria ser reduzido 5 vezes.
} 

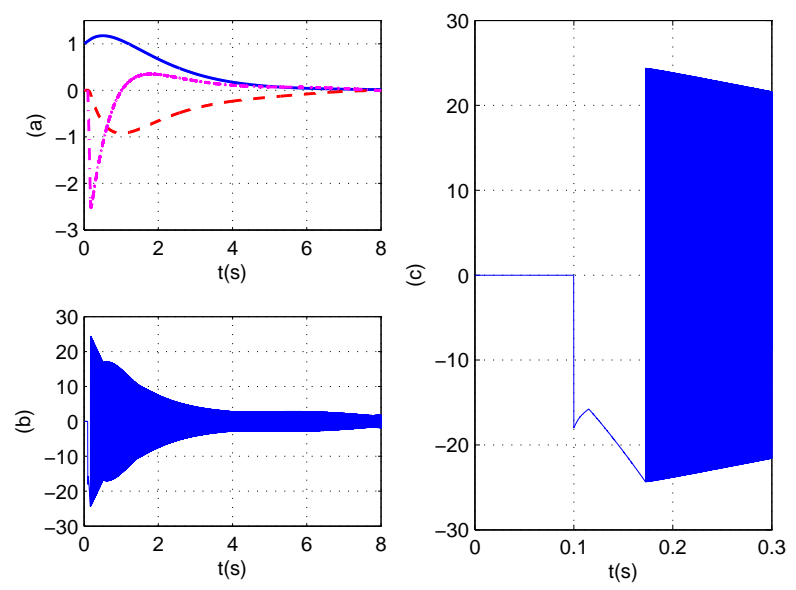

Figura 2: HGO e dwell-time: (a) estado $x_{e}$, (b) sinal de controle $u$ e (c) zoom no sinal de controle $u$ mostrando o dwell-time $\tau_{D}=0.1 \mathrm{~s}$.

considera-se $\xi_{1}(0)=2$ e $\mu=0.01$, enquanto que $u_{\text {sat }}=500$ na estratégia GBC.

Na Fig. $3\left(\right.$ a), pode-se observar que para $\xi_{1}(0)=2$, o desempenho de ambos os controladores é similar. No entanto, para condições iniciais menores $\left(\xi_{1}(0)=1\right.$ e 0.01$)$, uma degradação significativa da resposta transitória do erro de rastreamento é observada com a abordagem GBC comparada com a resposta com o esquema baseado em HGO e dwell-time $\left(\tau_{D}=0.1\right)$ ilustradas na Fig. $3(\mathrm{~b})-$ (c). Isso ocorre porque com o controlador proposto a magnitude de controle é automaticamente ajustada e torna-se menor para condições iniciais menores, resultando em um comportamento transitório mais uniforme em todo o domínio de atração. Por outro lado, na abordagem GBC, um domínio de atração maior requer um nível de saturação maior, assim como um ganho maior do HGO (parâmetro $\mu$ menor). Isso não evita os efeitos danosos do peaking, especialmente para condições iniciais pequenas, impedindo assim um comportamento transitório mais uniforme.

Uma vez que a estratégia de HGO e dwell-time não é baseada em saturação, o peaking é completamente evitado e o domínio de atração pode ser arbitrariamente aumentado reduzindo-se o parâmetro $\mu$ do HGO, sem prejudicar o comportamento transitório do rastreamento.

A estratégia baseada em HGO e dwell-time requer que detectemos qualquer instante de tempo em que a saída é desviada abruptamente de seu valor atual e reinicialize o controlador naquele instante. Se esse procedimento falhar, o peaking induzido no HGO pela mu-
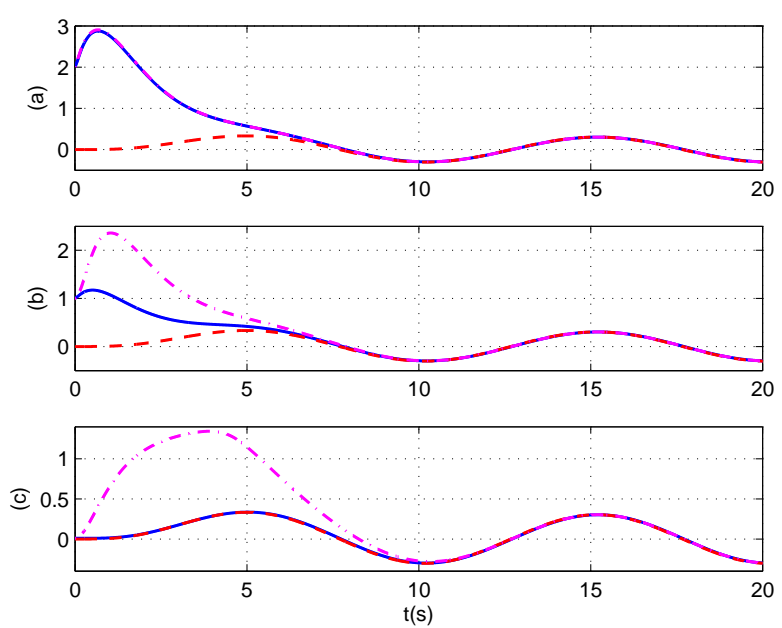

Figura 3: Transiente não-uniforme para condições iniciais diferentes: (a) $\xi_{1}(0)=2$, (b) $\xi_{1}(0)=1$ e (c) $\xi_{1}(0)=0.01$. Saída da planta $\xi_{1}=y$ (HGO e dwell-time: linha contínua; GBC: linha ponto-tracejada) e saída do modelo $y_{m}$ (linha tracejada).

dança repentina na saída leva o sistema à instabilidade. Essa situação pode ser observada quando escolhemos $\xi(0)=\left[\begin{array}{lll}0.5 & 0.5 & 0\end{array}\right]^{T}$ e inserimos pulsos de amplitude 0.15 e duração $0.0001 \mathrm{~s}$ na saída da planta $y$ nos instantes $t=1,2, \ldots, 5 \mathrm{~s}$. Essa perturbação é suficiente para induzir peaking nos estados do HGO e provocar escape em tempo finito em $t=1.06 \mathrm{~s}$ (curvas não mostradas). Por outro lado, a estratégia com dwell-time e esquema de monitoração (22)-(23) com $\alpha=2, \mu=0.001$ e $\tau_{D}=10 \mu$ lida eficientemente com as complicações causadas pelo peaking como mostrado na Fig. 4.

\section{Chattering no Sinal de Controle}

No Corolário 1, assegura-se que o deslizamento ideal remove o chattering no sinal de controle. Contudo, o deslizamento ideal não pode ser garantido em aplicações práticas pois os dispositivos reais de chaveamento sempre apresentam algum atraso ou limite na freqüência de chaveamento. Além disso, ruído, dinâmicas não-modeladas ou discretização estão sempre presentes. Sendo assim, como regra, o deslizamento ideal e o rastreamento perfeito não podem ser obtidos em aplicações reais. Nas simulações apresentadas (Figs. 2(b) e 4(b)), a lei de controle foi projetada com base na teoria. Entretanto, quando um dado controlador permite atingir o deslizamento ideal na ausência de tais "imperfeições", espera-se um desempenho prático melhor do que um outro controlador em que o deslizamento não é garantido 

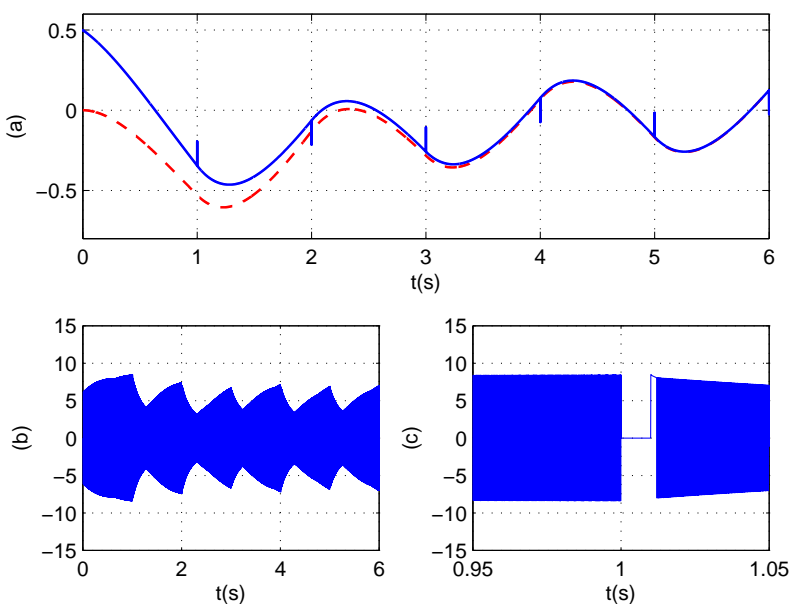

Figura 4: Desempenho do esquema de monitoração: (a) saída da planta $y$ corrompida por pulsos de perturbação (linha contínua) e saída do modelo de referência $y_{m}$ (linha tracejada), (b) sinal de controle $u$ e (c) zoom em $u$ mostrando a reinicialização do dwell-time devido ao pulso em $t=1 \mathrm{~s}$.

teoricamente. Além disso, o chattering no controle pode sempre ser reduzido ou eliminado por meio de estratégias adequadas, como por exemplo, o método da camada de fronteira apresentado em (Edwards \& Spurgeon, 1998).

\section{CONCLUSÕES}

O controlador por modos deslizantes e realimentação de saída para rastreamento de trajetórias de sistemas fortemente não-lineares incertos desenvolvido neste artigo utiliza as estimativas do HGO no cálculo da lei de chaveamento e na função de modulação (ganho de controle). Devido à estratégia de dwell-time na ativação do sinal de controle, o esquema proposto é livre de peaking. Foi provado que a abordagem proposta garante estabilidade exponencial semi-global (ou até global), com respeito a um pequeno conjunto residual independente das condições iniciais, sem a necessidade de fazer uso da saturação na lei de controle. Um esquema de monitoração foi também proposto para evitar o peaking induzido por classes de perturbações exógenas de saída.

\section{A DEMONSTRAÇÃO DO LEMA 1}

Introduzindo a transformação de coordenadas $\bar{x}_{e}=$ $T_{n} x_{e}$, com $T_{n}:=\left[\begin{array}{ll}I & S^{T}\end{array}\right]^{T}$ e $S$ definido em (9), o sistema (7) pode ser levado para a forma normal. Logo, pode-se concluir que (7) é OSS com respeito à saída de grau relativo unitário $S x_{e}$, ou seja, $x_{e}$ e $e$ satisfazem

$$
\left|x_{e}\right|,|e| \leq k_{1}\left|S x_{e}\right|+\pi_{1}
$$

com $k_{1}>0$ sendo uma constante e $\pi_{1}$ um termo exponencialmente decrescente que depende do valor do estado $x_{e}$ no início do intervalo $\mathcal{I}_{D}$. Note que, para todo $\tilde{x}_{e}$, ou $\left|S x_{e}\right| \leq\left|S \tilde{x}_{e}\right|$ ou $\left|S x_{e}\right|>\left|S \tilde{x}_{e}\right|$. Sendo assim, ou $\left|S x_{e}\right| \leq\left|S \tilde{x}_{e}\right|$ ou $\operatorname{sgn}(\hat{\sigma})=\operatorname{sgn}\left(S x_{e}\right)$. Considere o último caso.

Utilizando-se a função de energia $V=x_{e}^{T} P x_{e}$, onde $P=P^{T}>0$ é a solução de $A_{m}^{T} P+P A_{m}=-I$, pode-se assegurar que a derivada temporal de $V$ ao longo das soluções de (7) satisfaz $\dot{V} \leq-\left|x_{e}\right|^{2}-2 k_{p}\left|S x_{e}\right|[\varrho-|d|]$, ou, equivalentemente,

$$
\dot{V} \leq-\left|x_{e}\right|^{2}-2\left|S x_{e}\right|\left[\underline{k}_{p} \varrho-\left|k_{p} d\right|\right],
$$

onde $\underline{k}_{p}$ é o limitante inferior de $k_{p}$ definido em (H3). Desta forma, como $\varrho$ em (10) satisfaz (11), $\forall t \in \mathcal{I}_{D}$, tem-se que $\dot{V} \leq-\left|x_{e}\right|^{2}$, que juntamente com o caso $\left|S x_{e}\right| \leq\left|S \tilde{x}_{e}\right|$ leva à desigualdade $\left|S x_{e}\right| \leq\left|S \tilde{x}_{e}\right|+\pi_{2}$, onde $\pi_{2}$ é um termo exponencialmente decrescente. Conseqüentemente, a partir de (24), a dinâmica que governa $x_{e}$ é ISS com respeito a $\tilde{x}_{e}, \forall t \in \mathcal{I}_{D}$.

\section{B DEMONSTRAÇÃO DO TEOREMA 1}

Considere as definições apresentadas na Seção 6.1 para: o tempo de espera (dwell-time) $\tau_{D}$, o intervalo $\mathcal{I}_{D}:=$ $\left[\tau_{D}, t^{*}\right)$, o instante de tempo $t^{*} \in\left[\tau_{D}, t_{M}\right)$, o estado aumentado $x_{a}(t):=\left[\begin{array}{ll}x^{T} & \hat{\xi}^{T}\end{array}\right]^{T}$ e a bola $\mathcal{B}:=\left\{x_{a}:\left|x_{a}(t)\right| \leq\right.$ $R$ \} de raio $R>\left|x_{a}\left(\tau_{D}\right)\right|$.

\section{(A) Resultado Semi-Global}

Convergência e escape em tempo finito: A partir de (17), verifica-se que a desigualdade $\left|\tilde{x}_{e}\right| \leq \mathcal{O}(\mu)$ é valida $\forall t \in \mathcal{I}_{D}$ e $\mu$ suficientemente pequeno. Portanto, de acordo com o Lema 1, pode-se verificar diretamente que $x_{e}(t)$ e o estado completo do erro, $z(t)$ em (21), são limitados $\forall t \in \mathcal{I}_{D}$. Além disso, $z(t) \rightarrow \mathcal{O}(\mu)$ exponencialmente e não pode escapar em tempo finito, ou seja, $t^{*} \mathrm{e}$ $t_{M} \rightarrow+\infty$. Assim, a seguinte desigualdade se verifica:

$$
|z(t)| \leq \beta_{0}\left(\left|z\left(\tau_{D}\right)\right|\right) e^{-\lambda_{0}\left(t-\tau_{D}\right)}+\mathcal{O}(\mu), \forall t \geq \tau_{D},
$$

onde $\lambda_{0}>0$ é uma constante e $\beta_{0} \in \mathcal{K}_{\infty}$.

Estabilidade semi-global: Lembrando que o período de espera $\tau_{D}$ tende a zero quando $\mu \rightarrow 0$ e que $u=0$ $\forall t \in\left[0, \tau_{D}\right), \tau_{D}$ torna-se tão pequeno à medida que $\mu$ é reduzido de forma que a norma do estado da planta $|x|$, $\left|x_{e}\right|$ e $|z|$ permanecem próximos do conjunto de condições iniciais $\forall t \in\left[0, \tau_{D}\right)$. Portanto, pode-se considerar o 
intervalo $\forall t \in[0,+\infty)$ na desigualdade (25) e obter:

$$
|z(t)| \leq \bar{\beta}_{0}(|z(0)|) e^{-\underline{\lambda}_{0} t}+\mathcal{O}(\mu), \forall t,
$$

onde $\underline{\lambda}_{0}>0$ é uma constante e $\bar{\beta}_{0} \in \mathcal{K}_{\infty}$. Note que para $|z(0)|$ e $\mu$ suficientemente pequenos, tem-se que $|z(t)| \leq$ $R(\forall t)$. Além disso, como $R$ e $|z(0)|$ podem ser escolhidos arbitrariamente grandes quando $\mu \rightarrow 0$, a estabilidade exponencial semi-global do sistema do erro é obtida.

Sinais limitados: Relembrando que a norma de $\xi_{m}$ é uniformemente limitada, então $\xi=x_{e}+\xi_{m}$ também é uniformemente limitado e, a partir de (17) e (18), podese concluir que as normas de $\chi, x$ e todos os sinais em malha fechada são uniformemente limitadas.

Resíduo independente das condições iniciais: Note que o conjunto residual de ordem $\mathcal{O}(\mu)$ satisfaz $\mathcal{O}(\mu) \leq$ $k \mu$, onde a constante $k>0$ pode ser uma função das condições iniciais do sistema em malha fechada, devido à análise baseada na bola $\mathcal{B}$. Entretanto, para $\mu$ suficientemente pequeno e dependente das condições iniciais, o conjunto residual pode ser feito independente das condições iniciais. De fato, dada uma constante arbitrária $\bar{\mu}$, tem-se que $\mathcal{O}(\mu) \leq \mathcal{O}(\bar{\mu})$ para $\mu \leq \mathcal{O}(\bar{\mu}) / k$.

\section{(B) Resultado Global}

No caso semi-global anterior, a desigualdade (17) foi utilizada para obter uma função de modulação $\varrho$ livre de pico satisfazendo $\varrho \geq|d|$. Isso permitiu usar o Lema 1 para concluir o resultado semi-global. Por outro lado, a desigualdade (17) não pode ser utilizada diretamente para obtermos resultados globais, uma vez que esta é válida somente para $x_{a}$ dentro da bola $\mathcal{B}$.

Entretanto, se a dinâmica- $\eta$ for ausente ${ }^{2}$, o ganho de alta freqüência $k_{p}$ for uniformemente limitado $\left(\left|k_{p}\right| \leq \bar{k}_{p}\right.$, com $\bar{k}_{p}>0$ constante) e $d_{\phi}$ for globalmente Lipschitz (em (H5), $\varphi_{d}$ é linear), pode-se obter a equação do erro (7) com $d$ satisfazendo $\left|k_{p} d\right| \leq\left|k_{m}\right||r|+\left|K_{m}\right||\xi|+k_{0}|\xi|+k_{1}$, sendo $k_{0}$ e $k_{1}$ constantes positivas independente das condições iniciais (bola $\mathcal{B}$ ), tais que $\left|d_{\phi}\right| \leq k_{0}|\xi|+k_{1}$. Em outras palavras, neste caso encontra-se um limitante superior para a norma de $k_{p} d$ afim em $|\xi|$.

Além disso, pode-se escolher $\varrho$ de forma que $\left|\underline{k}_{p} \varrho\right|=k_{2}+$ $k_{3}|\hat{\xi}|$, com constantes $k_{2}, k_{3}$ satisfazendo $k_{2}>\left|k_{m}\right||r|+k_{1}$ e $k_{3} \geq\left|K_{m}\right|+k_{0}$. Portanto, lembrando que $\left|\tilde{x}_{e}\right|=|\xi-\hat{\xi}| \geq$ $|\xi|-|\hat{\xi}|$, a seguinte desigualdade pode ser verificada

$$
\underline{k}_{p} \varrho-\left|k_{p} d\right| \geq-k_{4}\left|\tilde{x}_{e}\right|
$$

onde $k_{4}$ é uma constante positiva.

\section{(1) Propriedade ISS de $\tilde{x}_{e}$ para $x_{e}$ :}

\footnotetext{
${ }^{2}$ Esta condição apenas simplifica o desenvolvimento, basta considerar $\varphi_{0} \equiv 0$ em (H4).
}

De (27) e seguindo a demonstração do Lema 1 para o caso em que $\left|S x_{e}\right|>\left|S \tilde{x}_{e}\right|$, pode-se verificar que a dinâmica de $x_{e}$ é ISS com respeito a $\tilde{x}_{e}, \forall t \in\left[\tau_{D}, t_{M}\right)$, uma vez que

$$
\dot{V} \leq-\left|x_{e}\right|^{2}+2 k_{4}\left|S x_{e}\right|\left|\tilde{x}_{e}\right|
$$

independentemente da desigualdade $\varrho \geq|d|$ ser verificada ou não. De fato, considere os seguintes casos: (i) $\left|x_{e}\right| \leq\left|\tilde{x}_{e}\right|\left(4 k_{4}|S|\right)$ ou (ii) $\left|x_{e}\right|>\left|\tilde{x}_{e}\right|\left(4 k_{4}|S|\right)$. Para o caso (ii), tem-se de (28) que $\dot{V} \leq-\lambda_{1} V$, onde $0<\lambda_{1}<1 / 2$ é uma constante apropriada. Portanto, utilizando a desigualdade de Rayleigh e considerando o caso (i), pode-se concluir que

$$
\left|x_{e}(t)\right| \leq \beta_{1}\left(\left|x_{e}(0)\right|\right) e^{-\lambda_{2} t}+k_{5}\left|\tilde{x}_{e}(t)\right|, \forall t \in\left[\tau_{D}, t_{M}\right),
$$

onde $\lambda_{2}, k_{5}>0$ são constantes e $\beta_{1} \in \mathcal{K}_{\infty}$.

\section{(2) Propriedade ISpS de $x_{e}$ para $\tilde{x}_{e}$ :}

Por outro lado, como será verificado a seguir, pode-se demonstrar a partir de (15) que a dinâmica que governa $\tilde{x}_{e}$ é ISpS com respeito a $x_{e}, \forall t \in\left[\tau_{D}, t_{M}\right)$, com ganhoISpS da ordem de $\mu$.

De fato, recordando que $\hat{\xi}=\xi-\tilde{x}_{e}, \tilde{x}_{e}=T_{\mu}^{-1} \zeta$ e $\xi=x_{e}+\xi_{m}$, pode-se reescrever $\hat{\xi}$ como $\hat{\xi}=-T_{\mu}^{-1} \zeta+x_{e}+\xi_{m}$. Além disso, como $\left|T_{\mu}^{-1}\right| \leq 1$, tem-se que $|\hat{\xi}| \leq|\zeta|+\left|x_{e}\right|+k_{6}$, onde $k_{6} \geq\left|\xi_{m}\right|$ é uma constante.

Portanto, pode-se obter um majorante afim em $\left|x_{e}\right|+|\zeta|$ para a função de modulação $\varrho$. Agora, considere o sinal $\nu$ dado em (15). Como $\left|d_{\phi}\right| \leq k_{0}|\xi|+k_{1}$ e $\bar{k}_{p} \geq\left|k_{p}\right| \geq \underline{k}_{p}>0$, pode-se escrever que $|\nu| \leq k_{7}|u| \leq k_{7} \varrho$ e também obter um majorante afim em $\left|x_{e}\right|+|\zeta|$ para a norma do sinal $\nu$ :

$$
|\nu| \leq k_{8}\left(\left|x_{e}\right|+|\zeta|\right)+k_{9}
$$

onde $k_{7}, k_{8}$ e $k_{9}$ são constantes positivas.

Considere agora a dinâmica de $\zeta$ descrita em (15) e a função de energia $V=\zeta^{T} P \zeta$, onde $P=P^{T}>0$ é a solução de $A_{o}^{T} P+P A_{o}=-I$. Então, a derivada temporal de $V$ ao longo das soluções de (15) satisfaz $\mu \dot{V}=-|\zeta|^{2}+(\mu \nu) k_{p}\left[2 \zeta^{T} P B_{\rho}\right]$ e, de $(30)$, tem-se que $\mu \dot{V} \leq-|\zeta|^{2}+\mathcal{O}(\mu)|\zeta|^{2}+\mathcal{O}(\mu)|\zeta|\left|x_{e}\right|+\mathcal{O}(\mu)|\zeta|$. Agora, considere dois casos: (i) $|\zeta|<4 \mathcal{O}(\mu)\left|x_{e}\right|+4 \mathcal{O}(\mu)$ ou (ii) $|\zeta| \geq 4 \mathcal{O}(\mu)\left|x_{e}\right|+4 \mathcal{O}(\mu)$. Para o caso (ii), tem-se que $\mathcal{O}(\mu)\left|x_{e}\right|, \mathcal{O}(\mu) \leq|\zeta| / 4$ e, portanto, a seguinte desigualdade se verifica: $\mu \dot{V} \leq-|\zeta|^{2}+\mathcal{O}(\mu)|\zeta|^{2}+|\zeta|^{2} / 4+|\zeta|^{2} / 4$, ou, equivalentemente,

$$
\mu \dot{V} \leq-[1 / 2-\mathcal{O}(\mu)]|\zeta|^{2}
$$

de onde pode-se concluir que $\mu \dot{V} \leq-\lambda_{3} V$, com uma constante apropriada $\lambda_{3}>0$ e $\mu$ suficientemente pequeno. 
Logo, considerando o caso (i) e utilizando a desigualdade de Rayleigh, pode-se concluir que

$\left|\tilde{x}_{e}\right| \leq \beta_{2}\left(\left|\tilde{x}_{e}(0)\right|\right) e^{-\lambda_{3} t}+\mathcal{O}(\mu)\left|x_{e}\right|+\mathcal{O}(\mu), \forall t \in\left[\tau_{D}, t_{M}\right)$,

onde $\beta_{2} \in \mathcal{K}_{\infty}$. Em (31), o limitante superior para a norma de $\tilde{x}_{e}$ foi obtido utilizando o fato de que $\tilde{x}_{e}=T_{\mu}^{-1} \zeta$ implica $\left|\tilde{x}_{e}\right| \leq|\zeta|$, pois $\left|T_{\mu}^{-1}\right| \leq 1$ para $\mu<1$. O resultado global pode ser obtido substituindo (31) em (29) e fazendo $\mu$ suficientemente pequeno (independente das condições iniciais). De fato, lembrando que o período de espera $\tau_{D}$ tende a zero quando $\mu \rightarrow 0$ e que $u=0$ $\forall t \in\left[0, \tau_{D}\right)$, pode-se considerar o intervalo $\forall t \in\left[0, t_{M}\right)$ nas desigualdades (29) e (31). Note que a análise acima está diretamente relacionada com o Teorema de Pequenos Ganhos de Jiang et al. (1994), entretanto aqui não utiliza-se a formulação com normas infinitas, sendo assim mais direta e menos conservadora.

Uma vez que $\mu$ é independente das condições iniciais do sistema em malha fechada, conseqüentemente, o conjunto residual de ordem $\mathcal{O}(\mu)$ também é independente. A prova de que todos os sinais no sistema de controle estão limitados e da não ocorrência de escape em tempo finito é idêntica à realizada no caso semi-global.

\section{DEMONSTRAÇÃO DO COROLÁRIO 1}

Relembrando-se que $A_{\rho}=A_{m}-B_{\rho} K_{m}, \hat{\xi}=\hat{x}_{e}+\xi_{m}$, $\hat{\xi}=x_{e}+\xi_{m}-\tilde{x}_{e}, \hat{x}_{e}=x_{e}-\tilde{x}_{e}$ e $\tilde{x}_{e}=T_{\mu}^{-1} \zeta$, pode-se verificar a partir de (13) que $\dot{\hat{x}}_{e}=A_{m} \hat{x}_{e}+B_{\rho} u+\varsigma_{m}+\varsigma_{e}$, onde $\varsigma_{m}=-B_{\rho}\left(K_{m} \xi_{m}+k_{m} r\right)$ e $\varsigma_{e}=\left(B_{\rho} K_{m}+H_{\mu} L_{o} C_{\rho}\right)\left(\tilde{x}_{e}-\right.$ $\left.x_{e}\right)+H_{\mu} L_{o} e$. Note que, de acordo com o Teorema 1 , todos os sinais do sistema em malha fechada são uniformemente limitados em norma e $z(t) \rightarrow \mathcal{O}(\mu)$. Então, existe um tempo finito $T_{1}>0$ tal que $\left|\varsigma_{e}\right| \leq \delta_{1}, \forall t \geq T_{1}$, e qualquer $\delta_{1}>0$. Agora, considere a função de energia $V=\hat{x}_{e}^{T} P \hat{x}_{e}$, onde $P=P^{T}>0$ é a solução de $A_{m}^{T} P+P A_{m}=-Q, \operatorname{com} Q=Q^{T}>0$ e $P B_{\rho}=S^{T}$ (lembrar que o sistema $\left(A_{m}, B_{\rho}, S\right)$ é estritamente real positivo). Então, calculando $\dot{V}$ ao longo das soluções da dinâmica que governa $\hat{x}_{e}$, pode-se verificar que a condição para a existência de modo deslizante $\hat{\sigma} \dot{\hat{\sigma}}<0$ é verificada para algum tempo finito $T_{2} \geq T_{1}$ desde que $\varrho \geq \varsigma_{m}+\delta$, onde $\delta>0$ é uma constante arbitrária.

\section{AGRADECIMENTOS}

Este trabalho contou com o apoio financeiro da FAPERJ e do CNPq. Os autores agradecem os comentários e as sugestões dos revisores anônimos.

\section{REFERÊNCIAS}

Andrieu, V. \& Praly, L. (2009). A unifying point of view on output feedback designs for global asymptotic stabilization, Automatica 45: 1789-1798.

Arcak, M. \& Kokotović, P. (2001). Observer-based control of systems with slope-restricted nonlinearities, IEEE Trans. Aut. Contr. 46(7): 1146-1150.

Boyd, S., El Ghaoui, L., Feron, E. \& Balakrishnan, V. (1994). Linear matrix inequalities in system and control theory, $1^{\text {st }}$ edn, SIAM.

Cunha, C. D., Araújo, A. D. \& Mota, F. C. (2009). Controlador em modo dual adaptativo robusto para plantas com grau relativo arbitrário, Controle $\&$ Automação 20: 72-82.

Cunha, J. P. V. S., Hsu, L., Costa, R. R. \& Lizarralde, F. (2005). Controle de sistemas lineares incertos por modos deslizantes e observador de alto ganho sem peaking, Controle \&̧ Automação 16(4): 449-466.

De Persis, C., De Santis, R. \& Morse, A. S. (2002). Nonlinear switched systems with state dependent dwelltime, In Proc. IEEE Conf. on Decision and Control, pp. 4419-4424.

Deaecto, G. S. \& Geromel, J. C. (2009). Controle de sistemas lineares com comutação, Controle \& $A u$ tomação 19: 431-443.

Edwards, C. \& Spurgeon, S. K. (1998). Sliding Mode Control: Theory and Applications, Taylor \& Francis Ltd.

El'youssef, E. S., Moreno, U. F., Castelan Neto, E. B. \& Pieri, E. R. (2007). A high-gain observer approach applied to the robust feedback linearization control, IFAC Symposium on System, Structure and Control, Foz do Iguaçu.

El'youssef, E. S., Pieri, E. R., Moreno, U. F. \& Castelan Neto, E. B. (2008). Realimentação linearizante com observadores de alto ganho: uma comparação, Congresso Brasileiro de Automática, Juiz de Fora.

Faria, F. A., Assunção, E. \& Teixeira, M. C. M. (2009). Realimentação da derivada dos estados em sistemas multivariáveis lineares usando LMIs, Controle E) Automação 20: 88-93.

Filippov, A. F. (1964). Differential equations with discontinuous right-hand side, American Math. Soc. Translations 42(2): 199-231. 
Freidovich, L. B. \& Khalil, H. K. (2007). Lyapunovbased switching control of nonlinear systems using high-gain observers, Automatica (43): 150-157.

Freidovich, L. B. \& Khalil, H. K. (2008). Performance recovery of feedback-linearization-based designs, IEEE Trans. Aut. Contr. 53(10): 2324-2334.

Hespanha, J. P., Liberzon, D. \& Morse, A. (2003). Overcoming the limitations of adaptive control by means of logic-based switching, Systems \& Contr. Letters 49: 49-65.

Hsu, L., Costa, R. R. \& Cunha, J. P. V. S. (2003). Modelreference output-feedback sliding mode controller for a class of multivariable nonlinear systems, Asian Journal of Control 5(4): 543-556.

Hsu, L., Cunha, J. P. V. S., Costa, R. R. \& Lizarralde, F. (2002). Multivariable output-feedback sliding mode control, in X. Yu \& J.-X. Xu (eds), Variable Structure Systems: Towards the 21st Century, SpringerVerlag, pp. 283-313.

Jiang, Z. P., Teel, A. R. \& Praly, L. (1994). Small-gain theorem for ISS systems and applications, Mathematics of Control, Signals and Systems (7): 95-120.

Kaliora, G., Astolfi, A. \& Praly, L. (2006). Norm estimators and global output feedback stabilization of nonlinear systems with ISS inverse dynamics, IEEE Trans. Aut. Contr. 51(3): 493-498.

Khalil, H. K. (2002). Nonlinear Systems, $3^{\text {nd }}$ edn, Prentice Hall.

Krichman, M., Sontag, E. D. \& Y., W. (2001). Inputoutput-to-state stability, SIAM J. Contr. Optim. 39(6): 1874-1928.

Krstić, M., Kanellakopoulos, I. \& Kokotović (eds) (1995). Nonlinear and adaptive control design, John Wiley \& Sons, Inc.

Li, S. \& Tao, G. (2009). Feedback based adaptive compensation of control system sensor uncertainties, Automatica 45: 393-404.

Marino, R. \& Tomei, P. (1995). Nonlinear Control Design. Geometric, Adaptive and Robust, $1^{\text {st }}$ edn, Prentice Hall.

Mazenc, F., Praly, L. \& Dayawansa, W. (1994). Global stabilization by output feedback: examples and counterexamples, Systems \& Contr. Letters 23: $119-125$.
Oh, S. \& Khalil, H. K. (1995). Output feedback stabilization using variable structure control, Int. J. Contr. 62(4): 831-848.

Oh, S. \& Khalil, H. K. (1997). Nonlinear outputfeedback tracking using high-gain observer and variable structure control, Automatica 33(10): 18451856.

Oliveira, T. R., Hsu, L. \& Peixoto, A. J. (2008a). Peaking free high gain observer based sliding mode control for uncertain systems with strong nonlinearities, Congresso Brasileiro de Automática, Juiz de Fora.

Oliveira, T. R., Hsu, L. \& Peixoto, A. J. (2008b). Peaking free output-feedback control of uncertain nonlinear systems, In Proc. American Contr. Conf., Seatlle, pp. 389-394.

Sontag, E. D. \& Y., W. (1997). Output-to-state stability and detectability of nonlinear systems, Systems 83 Contr. Letters 29: 279-290.

Sussmann, H. J. \& Kokotović, P. V. (1991). The peaking phenomenon and the global stabilization of nonlinear systems, IEEE Trans. Aut. Contr. 36(4): 424440.

Teel, A. \& Praly, L. (1995). Tools for semiglobal stabilization by partial state and output feedback, SIAM J. Contr. Optim. 33(5): 1443-1488.

Yin, G., Wang, L. Y. \& Kan, S. (2009). Tracking and identification of regime-switching systems using binary sensors, Automatica 45: 944-955. 